\title{
Numerical Simulation on Stress Measurement with Eddy Current Thermography
}

\author{
Shuwen $\mathrm{DENG}^{\mathrm{a}}$, Suixian $\mathrm{YANG}^{\mathrm{a}, 1}$,Yong $\mathrm{YAO}^{\mathrm{a}}$ \\ ${ }^{a}$ School of Mechanical Engineering, Sichuan University, Chengdu, China
}

\begin{abstract}
Stress in components will lead to the change of material properties and even failure. Therefore, the assessment for the stress state of components is play an important role in testing industry. As a non-contact and multi-physical field nondestructive testing method, eddy current thermography (ECT) can be applied to detect non-homogeneous electromagnetic characteristics parameter distribution in conductive materials. Internal stress and its distribution in a material will affect the value of electromagnetic characteristic parameters. If induction current applied on conductive material, the Joule's heat, which generate in the sample will lead to the temperature rise on the surface of the specimen by induction heating process. The temperature distribution on the specimen surface can be recorded by infra camera and stored as IR images or videos. The feature of the temperature distribution and its variation can be used to express the stress state in the specimen. It is concluded that there is an approximate linear relationship between the surface temperature appreciation and the loading force when the excitation source condition remains unchanged.
\end{abstract}

Keywords. Numerical simulation, stress measurement, eddy current thermography, conductive material

\section{Introduction}

The existence of residual stress in the structure is harmful to the safety of the structure in most cases. The detection and elimination of residual stress has always been a focus of industry. Real-time and accurate detection of residual stress in structures is an important means to ensure the safe operation of structures. The commonly used nondestructive testing methods for residual stress mainly include ultrasonic testing ${ }^{[1-3]}, \mathrm{X}$ ray diffraction testing ${ }^{[4]}$, neutron diffraction testing ${ }^{[5,6]}$ and magnetic testing ${ }^{[7-9]}$. Eddy current testing $^{[10-12]}$ and eddy current thermography testing residual stress are also extended based on magnetic measurement. According to the research status of the residual stress nondestructive testing (NDT) in China and abroad, the stress distribution of the structure can be obtained through the existing testing technology. However, the ultrasonic detection process needs coupling agent, the wave velocity is small and the influencing factors are many. X-ray method is harmful to human body and can only measure the stress in one direction at a time. Neutron diffraction measurement has a wide range of average stress, which is difficult to measure the gradient distribution of residual

${ }^{1}$ Corresponding Author, Suixian YANG, School of Mechanical Engineering, Sichuan University, Chengdu, China; E-mail: yangsx@163.com. 
stress precisely. The magnetic measurement method is limit to ferromagnetic materials with a small measurement range. Eddy current detection method has deficiency in detection range because of eddy current skin effect.

Compared to traditional stress measurement of nondestructive testing methods like ultrasonic etc., eddy current thermography method have abilities to obtain the noncontact testing result of a single large detection area; achieve high detection precision and resolution of complex geometry component; use the distribution of residual stresses for prior detection in a wide range. In recent years, the application of eddy current thermography detection method to stress detection technology are popularity ${ }^{[13-17]}$, many studies are focus on the stress distribution and temperature response curve in thermal image graph with different stress states and various conductive.

In [13], researchers obtained linear coupling relationship between stress and thermal conductivity by applying uniaxial tensile load on conductive material with eddy current thermography. In [14], tensile stress on the surface of specimen was preliminarily quantified by using eddy current thermography as well. In this paper, the influence of electrical conductivity, magnetic conductivity and thermal conductivity caused by different stress states on eddy current thermography testing results will be studied by numerical simulation.

\section{Methodology}

Electromagnetic parameters of conductive material are the link between ECT and stress detection. Result of stress measurement by using ECT is mainly affected by conductivity, magnetic permeability and thermal conductivity of the sample. The variation of conductivity and magnetic permeability mainly affect the induction heating stage of the testing process, while the variation of thermal conductivity mainly affects the cooling stage. The results depend on the heat propagation process and the eddy current distribution in the sample. Fig. 1 is the scheme of the testing principle.

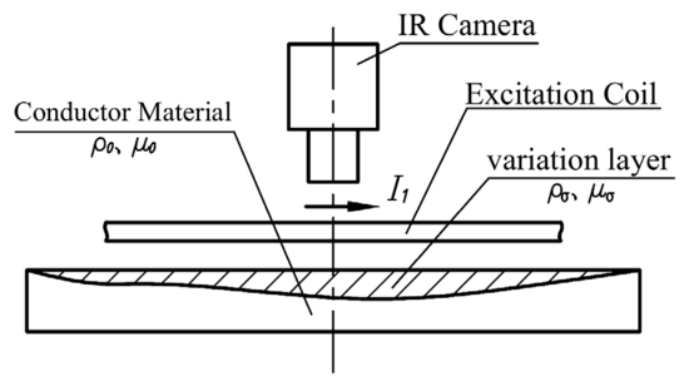

Fig. 1. Principle scheme of stress measurement with ECT.

During the testing process, the heat source of temperature rise is mainly provided by Joule's heating principle due to electromagnetic loss of conductivity material. If an alternating current with certain frequency applies on the excitation coil, there will be an electromagnetic induction phenomenon and eddy current of the same frequency will be generated in the sample ${ }^{[18]}$. The eddy current field in the sample is governed by Maxwell's equation as Eq.(1): 


$$
\nabla \times\left(\frac{1}{\mu} \nabla \times A\right)+\rho_{e} \frac{\partial A}{\partial t}+\varepsilon \frac{\partial^{2} A}{\partial t^{2}}=J
$$

Where $\mu$ is the magnetic permeability of the material, $A$ is the magnetic vector potential, $\rho_{e}$ is the conductivity of the material, $\varepsilon$ is the dielectric constant, and $J$ is the external current density.

\subsection{Coupling of solid mechanics and electromagnetic fields}

Metal resistance strain effect is the link between solid mechanics and electromagnetic field. The distance between atoms in the material and the dynamic distortion of the lattice will be changed by the internal stress of conductive material, which also lead to the change of the material's resistivity or the material's conductivity ${ }^{[19,20]}$. The conductivity is normally negative exponential with the change of stress. When the absolute value of the exponent is far less than 1, the high-order small term could be omitted, and the relationship between conductivity and stress as shown in Eq.(2) is approximately linear $^{[21]}$.

$$
\rho_{\sigma}=\rho_{0} \frac{1}{1+\alpha_{r} \sigma}
$$

Where, $\rho_{\sigma}$ is the electrical conductivity of metal with stress, $\rho_{0}$ is the electrical conductivity with no stress, $\sigma$ is the value of stress, and $\alpha_{r}$ is the stress coefficient.

\subsection{Coupling of electromagnetic field and temperature field}

The electromagnetic induction heating stage and the heat conduction stage are the connection between electromagnetic field and temperature field. According to Joule's law, part of the eddy current will be converted from electric energy to heat energy in the material. The generated heat $Q$ is proportional to the eddy current density $J_{s}$ and the electric field intensity $E$ as shown in Eq. (3).

$$
Q=J_{s} \cdot E \stackrel{J_{s}=\rho E}{\longrightarrow} Q=\frac{1}{\rho}\left|J_{s}\right|^{2}
$$

It can be seen from Eq.(2) that the Joule heat is mainly influenced by conductivity of material and eddy current density. The conductivity and magnetic permeability may also affect the value of eddy current density. The joule heat $Q$ generated in the induction heating stage will be transferred inside the tested piece, and the transfer rule is shown in Eq. (4).

$$
\rho C_{p} \frac{\partial T}{\partial t}-\nabla(\lambda \nabla T)=Q
$$


Where, $\rho$ is the material density, $C_{p}$ is the specific heat capacity of the material, $T$ is the thermodynamic temperature of the material, $\lambda$ is the thermal conductivity of the material.

\section{Methodology}

In this paper, a finite element model (FEM) for stress measurement with ECT is established on the COMSOL Multiphysics platform. Solid mechanics, electromagnetic field and temperature field are coupled in the model. The stress of the specimen is loaded with reference to the three-point bending experimental method in the solid mechanics module. During the loading process, compressive stress and tensile stress are respectively generated on the positive and negative surface of the specimen. The relationship between solid mechanics and electromagnetic field is established through the influence of stress on electrical conductivity, and the relationship between temperature field and electromagnetic field is established through joule thermal change. The load is set as a variable, and the law of the surface temperature changing with the load during the induction heating period is investigated. Accordingly, the relationship between the stress of the specimen and the surface temperature during the induction heating process can be derived.

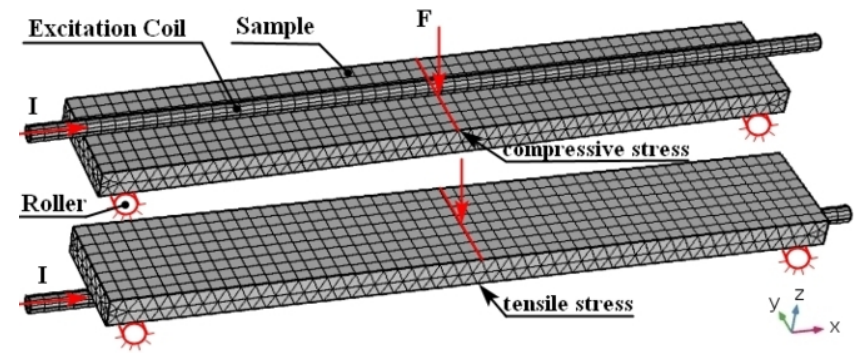

Figure 2. Geometry and parameters of the simulation model.

The simulation model of stress eddy current thermal imaging detection of conductor materials and its grid division are shown in Fig.2. The sample size is $260 \mathrm{~mm} \times 40 \mathrm{~mm} \times$ $10 \mathrm{~mm}$. The loading method of three-point bending mechanics experiment is adopted. The excitation coil is arranged $1 \mathrm{~mm}$ away from the surface of the specimen.

The electromagnetic parameters of the sample and the excitation parameters of the model are shown in Tab.1. The load $F$ gradually increases from 0 to $4000 N$ (the load of reference specimen is $O N$ ), and the direction of exciting coil is placed in parallel to the stress. The surface temperature of both sides of the three point bending specimens (the longitudinal displacement is negligible) are obtained by induction heating.

Table 1. The excitation parameters and electromagnetic parameters of sample

\begin{tabular}{cc}
\hline Parameter of sample & The value \\
\hline F & $0 \sim 4000 \mathrm{~N}$ \\
Young modulus & $2 \times 10^{11} \mathrm{~Pa}$ \\
Poisson's ratio & 0.3 \\
\hline I & $500 \mathrm{~A}$
\end{tabular}




\begin{tabular}{cc} 
frequency & $250 \mathrm{kHZ}$ \\
heating time & $500 \mathrm{~ms}$ \\
cooling time & $1000 \mathrm{~ms}$ \\
conductivity & $5 \times 10^{6} \mathrm{~S} / \mathrm{m}$ \\
thermal conductivity & $44.5 \mathrm{~W} /(\mathrm{m} \cdot \mathrm{K})$ \\
magnetic permeability & 1 \\
specific heat capacity & $475 \mathrm{~J} /(\mathrm{kg} \cdot \mathrm{K})$ \\
temperature coefficient of resistance & $1.23 \times 10^{-5} / \mathrm{K}$ \\
relative dielectric constant & 1 \\
density & $7850 \mathrm{~kg} \cdot \mathrm{m}^{-3}$ \\
\hline
\end{tabular}

\section{Results and discussions}

After coupling solid mechanics, electromagnetic field, temperature field and other physical fields with COMSOL simulation software, the simulation results are obtained as follows:

The surface plots of stress and surface temperature distribution on the specimens' surface after the three point bending experiment are shown in Fig.3 and Fig.4. It can be found that the specimen surface temperature of the compressive stress area is lower but that of the tensile stress area is higher than the same area of reference specimen. The larger the load is, the greater the temperature difference on the tensile stress side as well as on the compressive stress side is.

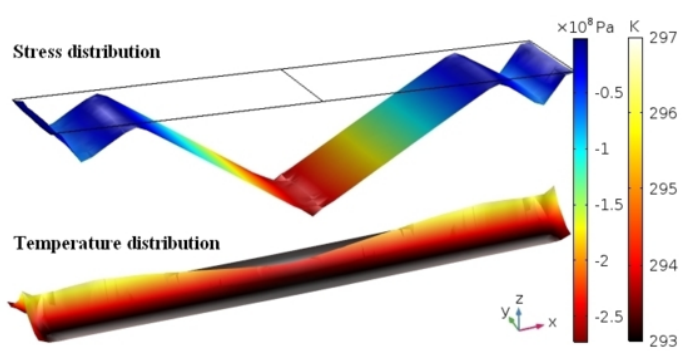

(a)

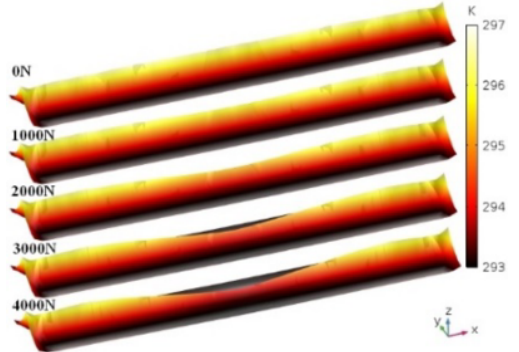

(b)

Fig. 3. Influence of load on temperature distribution of compressive stress side, (a) surface plots of stress and temperature distribution; (b) temperature distribution changes with load.

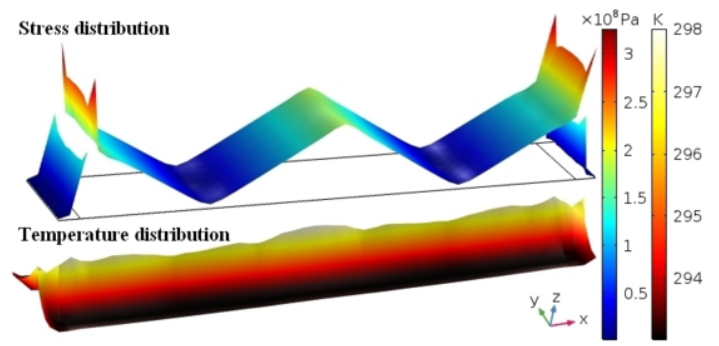

(a)

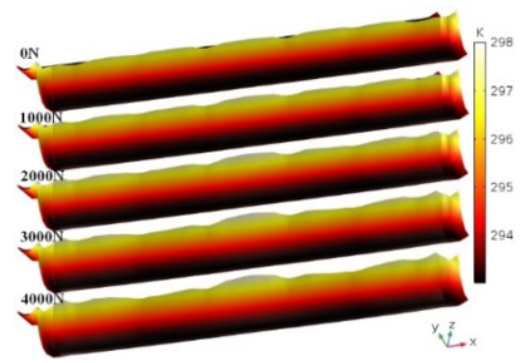

(b)

Fig. 4. Influence of load value on temperature distribution of tensile stress side, (a) surface plots of stress and temperature distribution; (b) temperature distribution changes with load. 
The curve relation between the load amplitude and the temperature appreciation is obtained by extracting the temperature value at the center of the simulation thermograph of the upper surface of the specimen and subtracting the temperature value of the unloaded specimen.

Fig.5 shows the relationship between temperature difference and load both on the central lines of the tensile stress side and compressive stress side along with X-axis. Lines in Fig.5 indicate that the surface temperature appreciation of the tensile stress side and compressive stress side has a positive and negative linear correlation with the load value respectively. Moreover, the further away the measuring point is from the centerline, the smaller the rate of temperature appreciation corresponding to this point changes with the loading force.

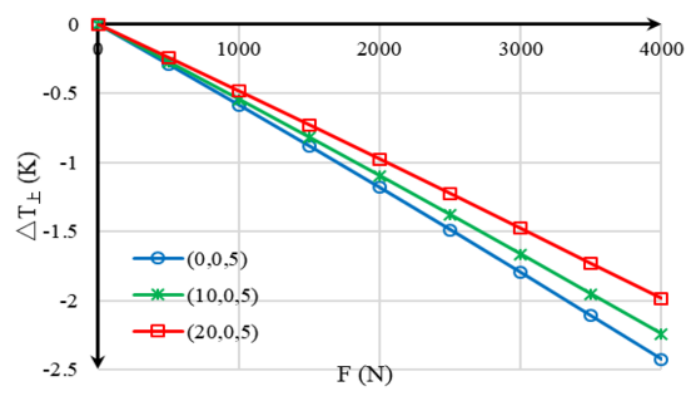

(a) compressive stress

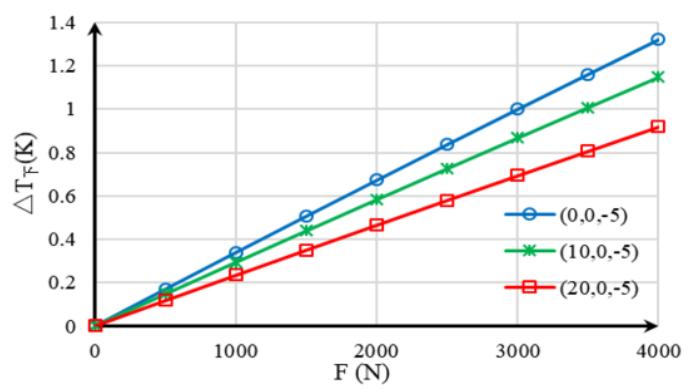

(b) tensile stress

Fig. 5. Effect of load value on temperature rise at different positions on specimen.

\section{Conclusions}

In this work, a simulation model coupled multi-physical fields has been established and the relationship between stress and temperature rise on the sample surface from the thermal images has been obtained by eddy current thermography. The results show that the stress value of the tested specimen has an approximate linear relationship with the temperature rise on the sample surface, in which the tensile stress is positively correlated with the temperature rise; meanwhile, the compressive stress is negatively correlated with the temperature rise. This result provides a theoretical foundation for the subsequent quantitative experimental study of residual stress. 
The residual stress measurement by using eddy current testing based on the correlation between stress and material conductivity has become a popularity subject in the field of nondestructive testing. However, it's still have several scientific problems need to be solved in the future.

- Three-dimensional nondestructive evaluation of stress tensor;

- Based on the electromagnetic non-destructive testing, the influence rule of the material's electromagnetic characteristics on the change of residual stress amplitude needs to be further studied;

- The mapping relationship between stress detection signals, stress amplitude and stress tensor based on eddy current method is needed to be revealed;

- The analysis technology of the corresponding relationship between residual stress state and eddy current thermal imaging test results (thermal imaging image sequence) needs to be further discussed;

- Residual stress detection system based on eddy current testing and the eddy current thermal image processing method have not been fully established.

In the future, we will research quantitative evaluation system for residual stress measurement by setting up the experimental platform by using eddy current thermal imaging test. Based on the theoretical and simulation research in this article, the plan for future experimental research work is as follows:

- The three-point bending experiment on residual stress of prefabricated specimens will be carried out, and the feasibility and accuracy of the experiment will be verified;

- Taking prefabricated sets of quantitative residual stress specimens as the test objects, eddy current thermal imaging experiments will be carried out;

- The eddy current thermographic test results corresponding to different residual stress specimens will be obtained, the data will be analyzed and the characteristic values for quantitative assessment of residual stress will be sought.

\section{References}

[1] Chunguang $\mathrm{Xu}$ et al. Nondestructive Testing Residual Stress Using Ultrasonic Critical Refracted Longitudinal Wave[J], Physics Procedia 70 (2015)594 - 598.

[2] W. Song, Q. Pan, C. Xu, X. Li and H. Liu, Benchmark of residual stress for ultrasonic nondestructive testing[C], 2013 Far East Forum on Nondestructive Evaluation/Testing: New Technology and Application, Jinan, 2013, pp. 73-76.,doi: 10.1109/FENDT.2013.6635532.

[3] W. Song et al., Residual stress nondestructive testing for pipe component based on ultrasonic method[C], 2014 IEEE Far East Forum on Nondestructive Evaluation/Testing, Chengdu, 2014, pp. 163-167.doi: 10.1109/FENDT.2014.6928254.

[4] GOU Guoqing, HUANG Nan, CHEN Hui et al. Detection of Residual Stress in Aluminum Alloy Carbody of High-Speed Train Using X-ray Diffraction Technology[J], Journal of Southwest Jiaotong University, 2012,47(4):618-622.

[5] Coules H E, Cozzolino L D, Colegrove P, et a1. Neutron diffraction analysis of complete residual stress tensors in conventional and rolled gas metal arc welds[J]. Experimen tal Mechanics, 2013, 53(2):195.

[6] Thibault D, Bocher P, Thomas $\mathrm{M}$, et at Residual stress characterization in Iow transformation temperature $13 \% \mathrm{Cr}-4 \% \mathrm{Ni}$ stainless steel weld by neutron diffraction and the contour method[J]. Material Sci. Eng. A, 2010, 527(23):6205.

[7] H. Ilker Yelbay et al. , Non-destructive determination of residual stress state in steel weldments by Magnetic Barkhausen Noise technique[J], NDT\&E International ,43(2010)29-33. 
[8] ZENG Jiewei SU Lanhai XU Liping et al. Research on the Stress Measurement of Steel Plate Based on Inverse Magnetostrictive Effect [J], Journal of Mechanical Engineering. 2014, 50(8):17-22.

[9] Blaszkiewicz, M., Albertin, L., \& Junker, W. (2009). The Eddy Current Technique for Determining Residual Stresses in Steels. Materials Science Forum, 210-213, 179-186. https://doi.org/10.4028/www.scientific.net/msf.210-213.179.

[10] Y. Shen, C. C. H. Lo, A. M. Frishman, et al., Conductivity profile determination by eddy current for shot-peened superalloy surfaces toward residual stress assessment, AIP Conference Proceedings 894, 1229 (2007); https://doi.org/10.1063/1.2718106.

[11] Y. Shen,C.C.H. Lo, N. Nakagawa, A.M. Frishman, Residual Stress Profile Assessment by Eddy Current for Shot Peened Nickel Superalloy[J]. Nondestruct Eval (2010) 29: 1-13.

[12] Haihong Huang, Cheng Yang, Zhengchun Qian, et al. Magnetic memory signals variation induced by applied magnetic field and static tensile stress in ferromagnetic steel[J]. Journal of Magnetism and Magnetic Materials, Volume 416, 15 October 2016, Pages 213-219.

[13] Xiaodong Zhou. Research and Detection of Residual Stress Based on PEC Thermal Imaging Technology[D]. University of Electronic Science and Technology of China, 2016.

[14] LEI Qing, LI Xuan, WANG Yahui. Research on Detection Method of Metal Structural Stress Based on Eddy Current Thermography[J]. Machine Design \& Research, 2017-(04):103-107.

[15] Urata S, Maeda Y, Nakai H. Classical Eddy Current Loss Evaluation of Electrical Steel Sheet under Compressive Stress[J]. TRANSACTIONS- INSTITUTE OF ELECTRICAL ENGINEERS OF JAPAN A, 2017, 137(10):577-583.

[16] Libing Bai, Gui Yun Tian, Stress Measurement Using Pulsed Eddy Current Thermography[C], 51st Annual conference of the British Institute of Non-Destructive Testing 2012, pp.462-473.

[17] LIU Luye, DENG Shuwen,YANG Suixian. Nondestructive Testing Technique for Residual Stress by Using Eddy Current Thermography and Its Application[J]. Nondestructive Testing, 2018, 40(10): 5-9.

[18] Mengchun Pan,Ze He, Dixiang Chen. Eddy Current Thermography Nondestructive Testing[M]. National Defense Industry Press, 2013.

[19] Xiaodong Zhou. Research and Detection of Residual Stress Based on PEC Thermal Imaging Technology[D]. University of Electronic Science and Technology of China, 2016.

[20] Zou Yu. Study on Eddy Current Nondestructive Testing of Dist-ributed Stress in Conductive Metal[D]. University of Electronic Science and Technology of China, 2016.

[21] REN Jilin, LIN Junming, XU Kebei. Eddy Current Testing[M]. China Mechine Press, 2013. 